\title{
FINAL REPORT FOR DOE GRANT NUMBER DE-FG03-88ER45378
}

\author{
Shechao Charles Feng - PI
}

February, 1995

\section{INTRODUCTION}

This is a final report on the above mentioned grant, entitled "applications of mesoscopic physics to novel correlations and fluctuations of speckle patterns: imaging and tomography with multiply scattered classical waves", which expired on September 14, 1994.

In section (2) below, we shall list the key research results we were able to obtain under the funding of this DOE grant, during the 1991-94 period. In section(3), we shall list all the publications that resulted from this DOE grant support.

\section{SUMMARY OF RESEARCH DURING THE 1991-94 FUNDING PERIOD}

In this section I shall summarize the highlights of our research activities during the current 1991-94 funding period which are funded by the DOE.

\subsection{Application of mesoscopic fiuctuations theory to correlations and fiuctuations of multiply scattered light}

One of the main areas I have been working on in the past three years was to apply the theoretical techniques first developed for "universal conductance fluctuations" theory to the problem of interference and correlations of classi- 


\section{DISCLAIMER}

Portions of this document may be illegible in electronic image products. Images are produced from the best available original document. 
cal wave propagation through multiple scattering media, such as when light propagates through milk or human tissues. In order to discuss our progress in this area, let us first summarize our earlier work on the application of mesoscopic conductance fluctuations theory to the problem of correlation in multiply scattered light, as the later work on imaging was based closely on this development.

Starting in 1987, one of my main research areas has been the application of the "universal conductance fluctuations" theory in mesoscopic conductors to the correlation properties of (classical) laser speckle patterns, which are the optical analog of electron wave interference patterns. In particular, my collaborators, Patrick Lee, Charlie Kane, and Doug Stone, and I discovered the novel "memory effect" for speckle pattern intensities generated from transmission of a laser beam through an elastic scattering medium, in the multiple scattering regime. To put simply, the memory effect $\left(C^{(1)}\right)$ dictates that even though a laser wave goes through a rather thick elastic scattering system, and therefore its wavefront is much distorted and seemingly random, it still "remembers" the wavefront of the incoming plane wave, so that as the incoming beam direction changes slightly, the transmitted speckle pattern will move accordingly[1]. This is a consequence of the non-self-averaging nature of coherent wave propagation through multiple elastic scattering systems. This result was regarded as a significantly new step forward in our understanding of speckle patterns in the multiple scattering regime, in that the conventional statistical theory of speckle patterns neglects all such correlation effects from the start. Our predictions of the memory effect were verified by subsequent experiments carried out at Bar-Mlan University in Israel, which I was also involved as an author[2]. We also predicted theoretically the interesting long range background correlations $\left(C^{(2)}\right)$ in the intensities of speckle patterns, as well as an "infinite range" correlations $\left(C^{(3)}\right)$ which are related to the universal conductance fluctuations. Our theory ties together previous disconnected pieces of theoretical approach to speckle correlations[3], showing how they can be fitted into the universal scheme of different correlation functions $\left(C^{(1)}\right.$, $C^{(2)}$, and $C^{(3)}$ ). The long range correlations $\left(C^{(2)}\right)$ has been experimentally tested by recent experiments performed in Prof. Lagendijk's group in Amsterdam, which shows excellent agreement with our theory [5], as well as experiments in Prof. Genack's group in Queen's college[6]. My student Lihong Wang and I later on generalized our theoretical studies of the three types of correlations for the reflection geometry, predicting similar effects as above[7]. Our work also ties in nicely with the recent subject of the so 
called "diffusing wave spectroscopy"[4], which has produced some amazing new experimental results in terms of being able to detect non-diffusive short time-scale motions of colloidal particles fluids[8].

My former postdoc Dr. Berkovits and I later proposed the idea that it may be possible to perform tomography or imaging with multiply scattered light or ultrasound [9]. A fixed scatterer or absorber in a fluid containing moving scatterers can induce a perturbed intensity peak in the near-field (time-averaged) transmitted or reflected intensity patterns which allows in principle determination of the position of this stationary scatterer or absorber, even though this object can be quite small. I am in the process of using this idea to attrick the applied problem of the possibility of detecting tumors in human tissues (a multiple scattering medium), using red or nearinfrared light. This study lead to significant application potentials in the field of medical optical imaging and characterization. Together with collaborator Didier Sornette and Christian Vanneste from Nice, France, I generalized the similar concept to formulate the principle of doing acoustic "non-destructive evaluations" of concrete or metal maeterials containing many cracks such that the transmitted acoustic waves are multiply scattered. By using the frequency average of the near field transmitted intensity for a given sample, we were able to show using extensive simulations that the position of a new crack can be found from the difference in near field speckle patterns[10].

In 1992, together with my former postdoc Richard Berkovitz, we studied the correlation properties of the optical analog of the disordered resonant tunneling device, namely the Fabry-Perot interferometer containing random scatterers. The main findings of our research on this topic is that the resonance peaks are broadened by disorder, and which depend separately on both the incoming and the outgoing-wave directions, unlike in the disorderfree case where the incoming and the outgoing wavevectors are identical due to translational symmetry. We also fount an enhanced narrow transmission peak due to weak localization effect in the mirror (or anti-specular) direction, whose height is exactly a factor of two. The width of this transmission weak localization peak is determined by the average path length inside the Fabry-Perot device, which can be much longer than the width of the device, if the mirrors have high reflection coefficients. We also calculated the $C^{(1)}$ correlation function for this device, as well as predicting the extreme sensitivity of the transmission intensity to the motion of a single scatterer inside the Fabry-Perot device[11]. 
Together with my former postdoc Leo Golubovic (who now teaches at West Virgina University), we worked on a variant of the light multiple scattering problem, namely the problem of classical and quantum superdiffusion in a time-dependent random potential. This problem can be considered as an interfering wave problem such that the wave propagation is directed along a special spatial direction, which we can consider as a fictitious "time". As is well known, a time independent random potential scatters a coherent wavefunction, whether it be electron wavefunction or light wavefunction, in such a way that leads to the effects of weak localization for weak disorder, and strong localization for strong disorder. The situation is very different when the random potential undergoes random temporal fluctuations. Physically, temporal fluctuations give rise to inelastic scattering for the quantum particle (or wavepacket), such that localization effects in fact are suppressed. This could lead to the "heating" of the quantum particle, such that if one places this particle at the origin at $t=0$, it can undergo super-diffusive behavior as a function of time. This is quite a striking effect to us. We made a systematic theoretical study of this problem, both analytically and numerically. We found that in fact it is possible to derive the exponents which govern such super-diffusive behavior for a quantum particle under the influence of a random potential that is fluctuating both in time and in space. Generally, in one dimension, we found that its ensemble averaged rms displacement $\delta r$ scales with time at long time in a continuum model as $\delta r \sim t^{6 / 5}$. We performed careful numerical simulations to verify this prediction. At dimensions $d \geq 2$, we found $\delta r \sim t^{9 / 8}$. These scaling laws are a result of the particle's kinetic energy increasing with time, as $E_{k} \sim t^{1 / 2}$ for $d \geq 2$ and $E_{k} \sim t^{2 / 5}$ in $d=1$. For lattice models, at sufficiently long times, the particle's kinetic energy will eventually saturate at the top of the band, such that the particle will eventually return to simple diffusion. Our work was published in the Physical Review Letters $(67,2115$ (1991)). We believe our general description of the bevarior of a quantum particle in a time-fluctuating random environment is an important new step in our search for better understanding of general wave phenomena in disordered systems.

Another recent progress we have been able to make in this area is to generalize our earlier work on correlation and fluctuation properties of multiply scattered light in linear optics to the corresponding transmission and reflection correlations of second harmonic waves in non-linear random Media. This work was done in collaboration with Prof. Lagendijk's group at the Unviersity of Amsterdam. I calculated analytically the $C^{(1)}$ correlation function 
for the non-linear speckle patterns, which was then successfully compared to the experimental measurements performed in Amsterdam. In a recently published Physical Review Letter[17], we presented both the theory and experiment of the $\left(C^{(\mathbf{1})}\right)$ correlations in both transmission and reflection geometries of second harmonic light generated inside a (powder) random medium, as a function of the angle of the incoming and outgoing light. The random medium consists of scattering particles with a non-linear susceptibility. We obtained the striking theoretical result, which are verified nicely by experiment, that correlations in reflection of the second harmonic light are mainly a function of the sample thickness $L$, in sharp contrast to the correlations in reflection of the fundamental light in the linear scattering regime, which mainly depend on the mean free path $l$. We also find the interesting result that memory effect in non-linear optics corresponds to $2 \Delta \theta_{\text {in }}=\Delta \theta_{\text {out }}$, where $\Delta \theta_{\text {in }}$ is the angular shift in the incoming fundamental light, and $\Delta \theta_{\text {out }}$ is that in the outgoing second-harmonic light.

\subsection{Quantum transport in localized electronic sys- tems}

Another main area we have been working hard on was the topic of interference effects in the localized regime. The interference effects on the metallic side of the metal-insulator transition are reasonaablly well understood now, after the development of weak localization theory and the theory of the universal conductance fluctuations. In comparison, the physics of interference effects on the localized side is much less understood. Thus it is natural for us to tackle several problems relating to interference effects in the localized regime, such as in a lightly doped semiconductor system.

In this effort, we first studied the problem of reproducible conductance fluctuations in macroscopic Anderson insulators, which was the analog problem of the universal conductance fluctuations in disordered metals. In a Physical Review Letter published in 1991[12], my collaborator Dr. JeanLouis Pichard from Saclay and I looked into the theoretical question as to how to estimate the magnetude of reproducible conductance fluctuations (the so called magneto-fingerprint) in such an insulator regime. Using the cur-

rent understanding in the fluctuations properties of an insulating system of size $L$ in the phase coherent regime from random matrix theories, namely $\left\langle[\delta \log (g)]^{2}\right\rangle=-\langle\log (g)\rangle$, where $g \sim \exp (-L / \xi)$ is the quantum conduc- 
tance of the system and $\xi$ is the localization length, we obtained an estimate that for a macroscopic localized system, $\delta G_{t o t} / G_{t o t}$ is $\sqrt{\frac{\xi_{p}^{D}}{\Omega_{e f f}}}$ for ergodic field changes $\Delta B \sim \phi_{0} / \xi^{2}$, where $\xi_{p}$ is a percolation correlation length which approaches macroscopic length scales at very low temperatures, and $\Omega_{\text {eff }}$ is an effective volume for the classical self-averaging of the fluctuations. Our theory shows reasonable agreement with recent experimental data on insulating systems in the Mott hopping regime.

I am continuing my research in this area with Dr. Pichard. We rccently considered the problem of conductance fluctuations in a localized system induced by the motion of a single impurity. We found a rather large effect, of order $\delta \log \left(g / g^{\prime}\right) \sim 1$ per phase coherent volume. This prediction could lead to interesting experiments and possible applications in the near future[13].

Another direction of our efforts in this area has been jointly performed with Prof. Boris Spivak, who visited my group in 1990-91 before joining the faculty at the University of Washington, namely the problem of negative magnetoresistance in variable range hopping conduction. Together with HuiLin Zhao, a former graduate of mine, we tried to clarify a central problem in this field: is there correction to the localization length $\xi$ due to magnetic field in localized systems, and at which dimensionalities? In a Physical Review B paper we published in 1991[14], we showed extensive numerical simulation results to illustrate that in two dimensions, there is indeed correction to the localization length as a function of the magnetic field $B$, such that a universal scaling relation can be established:

$$
L \equiv \ln \left(\frac{G(H)}{G(0)}\right)=\frac{\delta \xi(H)}{\xi^{2}} r_{m}=\gamma \frac{r_{m}}{L_{H}}
$$

where $G(H)$ is the system's conductance at a magnetic field $H, \gamma$ seems to be a nearly universal constant which does not seem to depend on details of the model, and $L_{H}$ is the magnetic length. This scaling law is valid as long as $\xi<L_{H}<r_{m}$, where $r_{m}$ is the Mott hopping distance which increases as temperatures are lowered. This striking prediction for the low field negative magnetoresistance contradict earlier predictions that Prof. Spivak himself and Prof. Shklovskii (from Leningrad, presently a professor at Minisota) made about ten years ago, which in fact were the standard theory in the field. Their old theory maintains that there is no correction to localization length, such that the maximum magnitude of the negative magnetoresistance is only of 
order a factor of two. Our new results indicate that in two dimensions, there is correction to localization length, such that the negative magnetoresistance can be an effect much larger than unity (i.e. $\ln [G(H) / G(0)] \gg 1$ ), provided the hopping length $r_{m}$ is sufficiently large. Our work has motivated much experimental activities. In fact, large negative magnetoresistance, of order a factor of eight, has been recently observed by Prof. Hong-Wen Jiang's group here at UCLA on insulating 2D electron gas systems[15] and Prof. Dahm's group (Case Western) [16]. These experimetal work seem to confirm our predictions, at least in semi-quantitative sense.

\subsection{Electron-phonon inelastic scattering rate and the temperature scaling exponent in integer quantum Hall effect}

Following a suggestion by Prof. Steve Kivelson, my student Hui-Lin Zhao and I studied in 1992 the problem of the mechanisms for inelastic scattering in the quantum Hall effect. Experiments carried out by Dan Tsui's group as well as in von Klitzing's group several years ago indicated that the width of the transition region between plateaus obeys scaling relations: temperature as $\Delta E \sim T^{\kappa}$, with $\kappa \approx 0.42$. This temperature scaling exponent was argued to be related to the inclastic scattering rate exponent $1 / \tau_{\text {in }} \sim T^{p}$ as $\kappa=p / 2 \mu$, where $\mu$ is the localization length exponent defined in $\xi_{\text {loc }} \sim \Delta E^{-\mu}$. Recent numerical simulations on short-range scattering potential models have yielded reliable values $\nu \approx 2.3-2.4$. Percolation arguments, which are valid for smooth potentials, in the semiclassical approximations gives $\mu=\nu+1=7 / 3$, where $\nu=4 / 3$ is the percolation length exponent $\left(\xi_{p} \sim \Delta E^{-\nu}\right)$, very close to the numerical findings for the Anderson model numerical work which models short-range disorder potential. For diffusive electron transport in zero field in $2 \mathrm{D}, p=1$ was found sometime ago for electron-electron scattering by Altshuler and Aronov. If one assumes that this value is still applicable at large field, one would be led to seeming disagreement with the experimental value for $\kappa$. Fermi liquid theory would give $p=2$, but it is valid only for ballistic transport regime, so that the factor of two should be absent in the relation between $\kappa$ and $p$. This again leads to difficulties in reconciling with the experimentally measured value for $\kappa$. We performed analytical calculations of the electron-phonon scattering rate in the semi-classical regime (smooth potential), and found that due to 
the high magnetic field, the linear regime $\left(1 / \tau_{\text {in }} \sim T\right)$ which normally applies only for $T>T_{D}$ (the Debye temperature) now moves all the way down to $T \approx \hbar c / l \approx 1 K$, where $l$ is the magnetic length. In contract, the electronelectron scattering rate is still $1 / \tau_{e c} \sim T^{2}$. This indicates that for a very wide temperature range in the smooth potential (percolation) regime, semiclassical analysis indicates that acoustic phonon scattering dominates the inelastic processes, and gives the value $p=1$. But because of the fractal nature of percolation perimeter paths for semi-classical electrons, the relation between the inelastic length and the inelastic scattering rate $1 / \tau_{\text {in }}$ is $L_{i n} \sim \tau_{i n}^{1 / D_{H}}$, where $D_{H}=7 / 4$ is the fractal dimension of the perimeter ("Hull") of the percolation clusters. In the semi-classical picture, the electron wavefunction decays only through tunneling events at the saddle points between percolation clusters, thus the correct crossover criterion between localized versus extended transport regimes is $L_{\text {in }} \approx \xi_{p}$, which separates the regime of transport where coherent, successive (sequential), tunneling dominates (the localized bahavior) and the regime where electron experiences de-phasing while orbiting on a percolation perimeter such that the sample's resistance is the classical addition of the various saddle point resistances (the extended behavior). These considerations lead to the result $\kappa=7 / 3$, as is found experimentally. We also have argument suggesting that this phoenomenon of the high temperature linear law for electron-phonon scattering rate being pushed down from $T_{D}$ to $\hbar c / l \sim 1 K$ is quite general, and in fact holds even for short range scattering potential. This can explain the seemingly universal value for $\kappa$ for both short-range scatterers and smooth random potentials. Our work on this subject has been published in the Physical Review Letters (70, 4134 (1993)).

\subsection{High frequency quantum transport in quantum well devices}

During the past three years, I have also been working on several applied physics topics relating to high frequency electron transport in quantum well and quantum point contact devices, motivated by experimental effort in making novel devices using the concepts of mesoscopic physics. I will list our results from these efforts briefly below.

1. Feasibility of far-infrared lasers using multiple semiconductor quantum wells: This work was motivated and performed together with my long time 
friend and colleague, Prof. Qing Hu, of the electrical engineering department at MIT. Prof. Hu is interested in making far-infrared lasers using quantum well structures, and posed to me various interesting theoretical questions. We have been looking at an important applied physics question: is it possible to make an infrared laser with adjustable frequencies based on inter-subband transitions in a quantum well? In an Applied Physics Letter we wrote in 1991[18], we made a preliminary feasibility analysis which seems to suggest that such a laser based on selective injection of electrons into an upper subband, and selective removal from a lower subband of a quantum well ising quantum-well energy filters, is quite possible. Photon confinement in our geometry is achieved between doped injector and collector semiconductor contacts which also serve as good photon reflectors. The lasing threshold current density is estimated by us to be in the range of $J_{t h} \sim 130 \mathrm{~A} / \mathrm{cm}^{2}$ at $5 T H z$.

We are still continuing to work in this technologically important area of research. With a graduate student Alexander Blank, we made the suggestion that an applied magnetic field, with its associated Landau quantization, can lead to a dramatic decrease of the electron-electron scattering non-radiative inter-subband transition rates, which tends to suppress lasing transitions; thus making it even more likely that a quantum well inter-subband laser can be fabricated and work. Our work on this topic has been published in the Journal of Applied Physics[19].

2. Far-infrared photon-assisted transport through quantum point contact devices: Another topic that is motivated from Prof. Hu's experimental work is the applicational possibilities of using quantum point contact devices as new far-infrared photon detectors. In a recent paper published in Physical Review $\mathrm{B}[20]$, we analyzed theoretically the phenomenon of photon-assisted quantum transport in split-gate quantum point contact devices. Both the transverse and longitudinal polarization configurations for the $\mathrm{AC}$ photon field were considered. We predicted that ministeps should appear in the drain/source conductance vs. gate voltage relation, as well as in the $I-V_{D S}$ curve in the nonlinear regime, for a quantum point contact irradiated with a coherent far-infrared radiation. The width of the ministeps is proportional to the radiation frequency, and the height of the ministeps is a function of the radiation power. We then calculated the current responsivity for this photon-assisted process in the limit of a small radiation signal, and showed that it is quite comparable to the quantum efficiency $e / \hbar \omega$ at and above 1 
$T H z$.

\section{List of publications in the 1991-94 funding period}

During the past three years, my group has produced a total of 19 publications, including 5 Physical Review Letters. These publications are listed below:

1. "Reproducible conductance fluctuations in macroscopic Anderson Insulators", Shechao Feng and J. L. Pichard, Phys. Rev. Lett. 67, 753 (1991).

2. "Classical and quantum superdiffusion in a time-dependent random potential", L. Golubovic, Shechao Feng, and Fan-an Zeng, Phys. Rev. Lett. 67,2115 (1991).

3. "Voltage fluctuations in mesoscopic conductors with single channel leads: electronic speckle patterns", Shechao Feng and B. Z. Spivak, Phys. Rev. B 44, 4014 (1991).

4. "Suppression of inter-edge-state equilibration due to multiple scattering with impurities", T. Martin and Shechao Feng, Phys. Rev. B 44, 9084 (1991).

5. "Negative magnetoresistance in variable range hopping conduction", H.-L. Zhao, B. Z. Spivak, M. P. Gelfand, and Shechao Feng, Phys. Rev. B 44, 10760 (1991).

6. "Feasibility of far-infrared lasers using multiple semiconductor quantum wells", Q. Hu and Shechao Feng, Appl. Phys. Lett. 59, 2923 (1991).

7. "Fabry-Perot interferometer with disorder: light localization and correlations", R. Berkovits and Shechao Feng, Phys. Rev. B 45, 97 (1992).

8. "Quantization effects on magnetoplasmons in quantum wells", L. Wang, F. Zeng, Shechao Feng, and Y. Zhu, Phys. Rev. B 46, 9804 (1992).

9. "Conductance of a quantum channel containing a small number of impurities", L. Wang, Shechao Feng, F. Zeng, and Y. Zhu, Phys. Lett. A 165,451 (1992).

10. "Anisotropic spectra of magnetoplasmons in wide parabolic quantumwell systems", L. Wang, Y. Zhu, F. Zeng, H. Zhao, and Shechao Feng, Phys. Rev. B 47, 16326 (1993). 
11. "Electron-phonon scattering rate in strong magnetic fields and the temperature scaling exponent between quantum Hall plateaus", H.-L. Zhao and Shechao Feng, Phys. Rev. Lett. 70, 4134 (1993).

12. "Conductance flucatuations due to motion of a single impurity in the localied regime", Shechao Feng, J.-L. Pichard, and F. Zeng, Phys. Rev. B 48,2529 (1993).

13. "Suppression of intersubband nonradiative transitions by a magnetic field in quantum well laser devices", A. Blank and Shechao Feng, J. of Appl. Phys. 74, 4795 (1993).

14. "Correspondance between correlation functions and enhanced backscattering peak for scattering from smooth random surfaces", A. Arsenieva and Shechao Feng, Phys. Rev. B 47, 13047 (1993).

15. "Far-infrared photon assisted transport through quantum point-contact devices", Shechao Feng and Q. Hu, Phys. Rev. B 48, 5354 (1993).

16. "Little-Parks and Aharonov-Bohm Oscillations in Fractional Hall Regime: Manifestation of Chern-Simons Gauge Flux", Shechao Feng and S.-C. Zhang, Phys. Rev. Lett. 71, 3533 (1993).

17. "Metal-insulator transitions between quantum Hall plateaus", H. L. Zhao and Shechao Feng, Physica A 200, 443 (1993).

18. "Non-destructive evaluations in multiple scattering media", C. Vanneste, Shechao Feng, and D. Sornette, Europhys. Lett. 24, 339 (1993).

19. "Transmission and reflection correlations of second harmonic waves in non-linear random media", J. de Boer, A. Lagendijk, R. Sprik, and Shechao Feng, Phys. Rev. Lett. 71, 3947 (1993). 


\section{References}

(1] S. Feng, C. Kane, P. A. Lee, and A. D. Stone, Phys. Rev. Lett. 61, 834 (1988); B. Shapiro, Phys. Rev. Lett. 57, 2168 (1986).

[2] I. Freund, M. Rosenbluh and S. Feng, Phys. Rev. Lett. 61, 2328 (1988).

[3] M. J. Stephen and G. Cwilich, Phys. Rev. Lett. 59, 285 (1987); P. A. Mello, E. Akkermans, and B. Shapiro, Phys. Rev. Lett. 61, 459 (1988).

[4] D. J. Pine, D. A. Weitz, P. M. Chaikin, and E. Herbolzheimer, Phys. Rev. Lett. 60, 1134 (1988); G. Maret and P. E. Wolf, Z. Phys. B 65, 409 (1987); M. J. Stephen, Phys. Rev. B 37, 1 (1988); A. Z. Genack, Phys. Rev. Lett. 58, 2043 (1987); see also D. J. Pine et al., in Scattering and Localization of Classical Waves in Random Media, edited by P. Sheng, (World Scientific, Singapore, 1990).

[5] M. P. van Albada, J. F. de Boer, and A. Lagendijk, Phys. Rev. Lett. 64, 2787 (1990).

[6] N. Garcia and A. Z. Genack, Phys. Rev. Lett. 63, 1678 (1989).

[7] L. Wang and S. Feng, Phys. Rev. B 40, 8283 (1989).

[8] S. Fraden and G. Maret, Phys. Rev. Lett. 65, 512 (1990); X. Qiu, X. L. Wu, J. Z. Xue, D. J. Pine, D. A. Weitz, and P. M. Chaikin, Phys. Rev. Lett. $65,516(1990)$.

[9] R. Berkovits and S. Feng, Phys. Rev. Lett. 65, 3120 (1990).

[10] C. Vanneste, Shechao Feng, and D. Sornette, Europhys. Lett. 24, 339 (1993).

[11] R. Berkovits and S. Feng, Phys. Rev. B 45, 97 (1992).

[12] S. Feng and J.-L. Pichard, Phys. Rev. Lett. 67, 753 (1991).

[13] S. Feng, J.-L. Pichard, and F. Zeng, Phys. Rev. B 48, 2529 (1993).

[14] H. Zhao, B. Spivak, M. Gelfand, and S. Feng, Phys. Rev. B 44, 10760 (1991). 
[15] H. W. Jiang, C. E. Johnson, and K. L. Wang, Phys. Rev. B 46, 12830 (1992).

[16] Y. Carmi, X. L. Hu, A. J. Dahm, and H. W. Jiang, in proceedings of LT20 in Portland, Oregon, 1993.

[17] J. de Boer, A. Lagendijk, R. Sprik, and S. Feng, Phys. Rev. Lett. 71, 3947 (1993).

[18] Q. Hu and S. Feng, Appl. Phys. Lett. 59, 2923 (1991).

[19] A. Blank and S. Feng, J. Appl. Phys. 74, 4795 (1993).

[20] S. Feng and Q. Hu, Phys. Rev. B 48, 5354 (1993).

\section{DISCLAIMER}

This report was prepared as an account of work sponsored by an agency of the United States Government. Neither the United States Government nor any agency thereof, nor any of their employees, makes any warranty, express or implied, or assumes any legal liability or responsibility for the accuracy, completeness, or usefulness of any information, apparatus, product, or process disclosed, or represents that its use would not infringe privately owned rights. Reference herein to any specific commercial product, process, or service by trade name, trademark, manufacturer, or otherwise does not necessarily constitute or imply its endorsement, recommendation, or favoring by the United States Government or any agency thereof. The views and opinions of authors expressed herein do not necessarily state or reflect those of the United States Government or any agency thereof. 